\title{
A NOTE ON AUTOMORPHISMS OF THE AFFINE CREMONA GROUP
}

\author{
IMMANUEL STAMPFLI
}

\begin{abstract}
Let $\mathcal{G}$ be an ind-group and let $\mathcal{U} \subseteq \mathcal{G}$ be a unipotent ind-subgroup. We prove that an abstract group automorphism $\theta: \mathcal{G} \rightarrow \mathcal{G}$ maps $\mathcal{U}$ isomorphically onto a unipotent ind-subgroup of $\mathcal{G}$, provided that $\theta$ fixes a closed torus $T \subseteq \mathcal{G}$, which normalizes $\mathcal{U}$ and the action of $T$ on $\mathcal{U}$ by conjugation fixes only the neutral element. As an application we generalize a result by Hanspeter Kraft and the author as follows: If an abstract group automorphism of the affine Cremona group $\mathcal{G}_{3}$ in dimension 3 fixes the subgroup of tame automorphisms $T \mathcal{G}_{3}$, then it also fixes a whole family of non-tame automorphisms (including the Nagata automorphism).
\end{abstract}

\section{Introduction}

Throughout this note we denote by $\mathcal{G}_{n}$ the group of polynomial automorphisms $\operatorname{Aut}\left(\mathbb{A}^{n}\right)$ of the complex affine space $\mathbb{A}^{n}=\mathbb{C}^{n}$. Such an automorphism has the form $\mathbf{g}=\left(g_{1}, \ldots, g_{n}\right) \in \mathcal{G}_{n}$ with polynomials $g_{1}, \ldots, g_{n} \in \mathbb{C}\left[x_{1}, \ldots, x_{n}\right]$. We define $\operatorname{deg} \mathbf{g}:=\max _{i} \operatorname{deg} g_{i}$. The tame automorphism group $T \mathcal{G}_{n}$ is the subgroup of $\mathcal{G}_{n}$ generated by the affine linear automorphisms, (i.e., the automorphisms $\mathbf{g}$ with $\operatorname{deg} \mathbf{g} \leq 1)$ and the triangular automorphisms, (i.e., the automorphisms $\left(g_{1}, \ldots, g_{n}\right)$ where $g_{i}=g_{i}\left(x_{i}, \ldots, x_{n}\right)$ depends only on $x_{i}, \ldots, x_{n}$ for each $\left.i\right)$. The main result of Hanspeter Kraft and the author [7] is the following.

Theorem 1. Let $\theta: \mathcal{G}_{n} \rightarrow \mathcal{G}_{n}$ be an abstract automorphism. Then there exist $\mathbf{g} \in \mathcal{G}_{n}$ and a field automorphism $\tau: \mathbb{C} \rightarrow \mathbb{C}$ such that

$$
\theta(\mathbf{f})=\tau\left(\mathbf{g} \circ \mathbf{f} \circ \mathbf{g}^{-1}\right) \quad \text { for all tame automorphisms } \mathbf{f} \in T \mathcal{G}_{n} .
$$

If $\theta$ preserves in addition the ind-group structure of $\mathcal{G}_{n}$ (see below for a definition), then Alexei Belov-Kanel and Jie-Tai Yu proved recently that $\theta$ is an inner automorphism of $\mathcal{G}_{n}$ (see [3]).

In dimension $n=2$ all automorphisms are tame (cf. [6,11]). But in dimension $n=3$, Ivan P. Shestakov and Ualbai U. Umirbaev showed that the famous Nagata automorphism $\mathbf{u}_{N} \in \mathcal{G}_{3}$ (see below for a definition) is non-tame (cf. [10]). It is an open problem if there exist non-tame automorphisms in dimension $n>3$. A natural question is, whether Theorem 1 extends to the entire automorphism group $\mathcal{G}_{n}$, i.e., whether $\theta(\mathbf{f})=\tau(\mathbf{g} \circ \mathbf{f} \circ \mathbf{g})$ for all $\mathbf{f} \in \mathcal{G}_{n}$. If this would be true, then every abstract automorphism of $\mathcal{G}_{n}$ would preserve the algebraic subgroups of $\mathcal{G}_{n}$ (see below for a definition). In fact, a main tool in the proof of Theorem 1 is to show that certain algebraic subgroups are sent to isomorphic algebraic subgroups under an abstract automorphism of $\mathcal{G}_{n}$. The main point of this note is to refine these techniques. In

Received by the editors November 16, 2012. 
order to state the main result, we introduce the concept of an ind-group and related terms.

A group $\mathcal{G}$ is called an ind-group if it is endowed with a filtration by affine varieties $G_{1} \subseteq G_{2} \subseteq \ldots$, each one closed in the next, such that $\mathcal{G}=\bigcup_{i=1}^{\infty} G_{i}$ and such that the map $\mathcal{G} \times \mathcal{G} \rightarrow \mathcal{G},(x, y) \mapsto x \cdot y^{-1}$ is a morphism of ind-varieties (see [8, chapter IV] for an introduction to ind-varieties and ind-groups). We then write $\mathcal{G}=\underline{\lim } G_{i}$. For example, $\mathcal{G}_{n}=\lim G_{n, i}$ is an ind-group, where $G_{n, i}$ is the set of all automorphisms $\mathbf{g} \in \mathcal{G}_{n}$ with $\operatorname{deg} \mathbf{g} \leq i$ (see [2]). We endow an ind-group $\mathcal{G}=\underline{\lim } G_{i}$ with the following topology: a subset $X \subseteq \mathcal{G}$ is closed if and only if $X \cap G_{i}$ is closed in $G_{i}$ with respect to the Zariski topology for each $i$. If $\mathcal{H} \subseteq \mathcal{G}$ is a closed subgroup, then $\mathcal{H}$ inherits in a canonical way an ind-structure from $\mathcal{G}$, namely $\mathcal{H}=\lim \mathcal{H} \cap G_{i}$. But for our purposes, we need a more general definition of an ind-subgroup.

Definition 1. Let $\mathcal{H}$ be a subgroup of an ind-group $\mathcal{G}=\lim G_{i}$. We say that $\mathcal{H}$ is an ind-subgroup of $\mathcal{G}$ if $\mathcal{H}$ can be turned into an ind-group $\overrightarrow{\mathcal{H}}=\underline{\lim } H_{k}$ such that to every $k$ there exists $i=i(k)$ such that $H_{k} \subseteq G_{i}$ is closed. Clearly, the ind-structure of $\mathcal{H}$ is then unique. We say that $\mathcal{H}$ is an algebraic subgroup of $\mathcal{G}$, if $\mathcal{H}$ is closed in $\mathcal{G}$ and contained in some $G_{i}$.

Definition 2. We say that an ind-group $\mathcal{U}$ is unipotent if $\mathcal{U}=\underline{\lim } U_{i}$ where $U_{i}$ is a unipotent algebraic group for all $i$.

Remark 1. Every element in a unipotent ind-group is unipotent. We do not know whether an ind-group consisting only of unipotent elements is always unipotent. If the ind-group is commutative, then we are able to prove this.

Main Theorem. Let $\theta: \mathcal{G} \rightarrow \mathcal{G}$ be an abstract automorphism of an ind-group $\mathcal{G}$ that is the identity on a closed torus $T \subseteq \mathcal{G}$. If $\mathcal{U} \subseteq \mathcal{G}$ is a unipotent ind-subgroup that is normalized by $T$ and if the neutral element of $\mathcal{U}$ is the only element that is fixed under conjugation by $T$, then $\theta(\mathcal{U})$ is a unipotent ind-subgroup of $\mathcal{G}$ and $\left.\theta\right|_{\mathcal{U}}: \mathcal{U} \rightarrow \theta(\mathcal{U})$ is an isomorphism of ind-groups.

Recall that there exists a bijective correspondence between locally nilpotent derivations of $\mathbb{C}\left[x_{1}, \ldots, x_{n}\right]$ and unipotent elements of $\mathcal{G}_{n}$, given by $D \mapsto \exp (D)$ where

$$
\exp (D)=\left(\sum_{i=0}^{\infty} \frac{D^{i}\left(x_{1}\right)}{i !}, \ldots, \sum_{i=0}^{\infty} \frac{D^{i}\left(x_{n}\right)}{i !}\right)
$$

(see [4, Section 1.5]). If $D$ is a locally nilpotent derivation and $f \in \operatorname{ker} D$ then $f D$ is again a locally nilpotent derivation and we call $\exp (f D)$ a modification of $\exp (D)$. For example, the Nagata automorphism $\mathbf{u}_{N}$ is a modification of $\mathbf{u}:=\exp D$ where

$$
D=-2 y \frac{\partial}{\partial x}+z \frac{\partial}{\partial y}, \quad p=x z+y^{2} \in \operatorname{ker} D \quad \text { and } \quad \mathbf{u}_{N}=\exp (p D) .
$$

Recently, Shigeru Kuroda gave a characterization of the non-tame modifications of certain unipotent automorphisms (see [9, Theorem 2.3]). This result implies that for all $f \in \operatorname{ker} D \backslash \mathbb{C}[z]$ the modification $\exp (f D)$ of $\mathbf{u}$ is non-tame. Clearly, all the modifications of $\mathbf{u}$ lie in the centralizer $\operatorname{Cent}(\mathbf{u})$. As a consequence of our Main Theorem we get the following result. 
Application. Let $\theta: \mathcal{G}_{3} \rightarrow \mathcal{G}_{3}$ be an abstract automorphism that is the identity on the tame automorphisms $T_{\mathcal{G}_{3}}$. Then $\theta$ fixes $\operatorname{Cent}(\mathbf{u})$ where $\mathbf{u}=\exp (D)$ and $D=$ $-2 y \cdot \partial / \partial x+z \cdot \partial / \partial y$. In particular, $\theta$ fixes the non-tame automorphisms $\exp (f D)$ where $f \in \operatorname{ker} D \backslash \mathbb{C}[z]$ and thus $\theta$ fixes the Nagata automorphism $\mathbf{u}_{N}$.

Remark 2. All the results and proves work over any uncountable algebraically closed field of characteristic zero.

1. Proof of the Main Theorem. Let $V$ be a commutative unipotent algebraic group. Recall that $V$ has a unique $\mathbb{C}$-vector space structure such that the product in $V$ corresponds to addition. Also recall that a map of commutative unipotent algebraic groups $V \rightarrow V^{\prime}$ is a homomorphism of algebraic groups if and only if it is $\mathbb{C}$-linear.

We start with a lemma that proves the Main Theorem in the case when $\mathcal{U} \subseteq \mathcal{G}$ is an algebraic subgroup isomorphic to $\mathbb{C}^{+}$.

Lemma 1. Let $\theta: \mathcal{G} \rightarrow \mathcal{G}$ be an abstract automorphism that is the identity on a closed torus $T \subseteq \mathcal{G}$ and let $U \subseteq \mathcal{G}$ be an algebraic subgroup isomorphic to $\mathbb{C}^{+}$ which is normalized by $T$ with character $\lambda$. If $\lambda$ is non-trivial, then $\theta(U) \subseteq \mathcal{G}$ is an algebraic subgroup isomorphic to $\mathbb{C}^{+}$and $T$ normalizes $\theta(U)$ with the same character $\lambda$. Moreover, $\left.\theta\right|_{U}: U \rightarrow \theta(U)$ is an isomorphism of algebraic groups.

Proof. Let $U^{\prime}:=\theta(U) \subseteq \mathcal{G}$. Choose $\mathbf{u}_{0} \in U$ that is different from the neutral element $\mathbf{e} \in \mathcal{G}$. Then, $U^{\prime} \backslash\{\mathbf{e}\}=\left\{\mathbf{t} \cdot \theta\left(\mathbf{u}_{0}\right) \cdot \mathbf{t}^{-1} \mid \mathbf{t} \in T\right\}$ and $\{\mathbf{e}\}$ are constructible subsets of some filter set of $\mathcal{G}$ and since $U^{\prime}$ is a group it follows that $U^{\prime} \subseteq \mathcal{G}$ is an algebraic subgroup (see $\left[5,7.4\right.$ Proposition A]). Since $U^{\prime}$ has no element $\neq \mathbf{e}$ of finite order, $U^{\prime}$ is unipotent. As $U^{\prime}$ is a toric variety with exactly two orbits, $U^{\prime}$ is one-dimensional (see also [7, Proposition 2]). Let $\lambda^{\prime}$ be the character of $U^{\prime}$. We have

$$
\theta\left(\lambda(\mathbf{t}) \mathbf{u}_{0}\right)=\theta\left(\mathbf{t} \cdot \mathbf{u}_{0} \cdot \mathbf{t}^{-1}\right)=\mathbf{t} \cdot \theta\left(\mathbf{u}_{0}\right) \cdot \mathbf{t}^{-1}=\lambda^{\prime}(\mathbf{t}) \theta\left(\mathbf{u}_{0}\right) \quad \text { for all } \mathbf{t} \in T .
$$

Hence, it follows that $\lambda$ and $\lambda^{\prime}$ have the same kernel and thus $\lambda= \pm \lambda^{\prime}$. If we take $\mathbf{t} \in T$ such that $\lambda(\mathbf{t})=2$ then equation $(*)$ implies that $\lambda^{\prime} \neq-\lambda$. Hence, $\lambda=\lambda^{\prime}$ and $\left.\theta\right|_{U}: U \rightarrow U^{\prime}$ is $\mathbb{C}$-linear by equation $(*)$.

Proof of the Main Theorem. Let $U \subseteq \mathcal{U}$ be an algebraic subgroup that is normalized by $T$. Choose closed algebraic subgroups $V_{1}, \ldots, V_{r} \subseteq U$ which are isomorphic to $\mathbb{C}^{+}$ and which are normalized by $T$, such that Lie $U=\operatorname{Lie} V_{1} \oplus \cdots \oplus \operatorname{Lie} V_{r}$. Thus, for suitable indices $i_{1}, \ldots, i_{m}$ we have $U=V_{i_{1}} \cdots V_{i_{m}}$ (see [5, 7.5 Proposition]). According to Lemma $1, \theta(U)=\theta\left(V_{i_{1}}\right) \cdots \theta\left(V_{i_{m}}\right)$ is a constructible subset of some $G_{i} \subseteq \mathcal{G}$ and thus $\theta(U)$ is an algebraic subgroup of $\mathcal{G}$. Again, since no element $\neq \mathbf{e}$ in $U$ has finite order, $\theta(U)$ is unipotent. Consider the following commutative diagram.

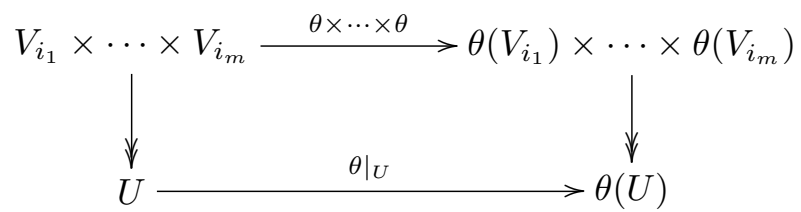

The vertical maps are induced by the product in $\mathcal{U}$ and hence they are surjective morphisms. The top horizontal map is an isomorphism of varieties by Lemma 1 . The lemma below due to Hanspeter Kraft implies that the abstract group homomorphism $\left.\theta\right|_{U}$ is an isomorphism of algebraic groups. 
As we can replace the filtration of $\mathcal{U}$ by a filtration of unipotent algebraic subgroups, each one normalized by $T$, it follows that $\theta(\mathcal{U}) \subseteq \mathcal{G}$ is a unipotent ind-subgroup and $\left.\theta\right|_{\mathcal{U}}: \mathcal{U} \rightarrow \theta(\mathcal{U})$ is an isomorphism of ind-groups.

Lemma 2 (Hanspeter Kraft). Let $X$ and $Y$ be affine varieties and let $f: X \rightarrow Y$ be an abstract map. If there exists a surjective morphism $g: Z \rightarrow X$ such that the composition $f \circ g: Z \rightarrow Y$ is a morphism and if $X$ is normal, then $f$ is a morphism.

For the proof of this lemma, one shows that the graph of $f$ is closed in $X \times Y$.

2. Proof of the Application. First, we determine the structure of Cent $(\mathbf{u})$. Denote by $E$ the partial derivative with respect to $x$. The ind-subgroups of $\mathcal{G}_{3}$ listed below are clearly contained in $\operatorname{Cent}(\mathbf{u})$.

$$
\begin{aligned}
C & :=\left\{(a x, a y, a z) \mid a \in \mathbb{C}^{*}\right\}, \\
\mathcal{F} & :=\{\exp (f D) \mid f \in \operatorname{ker} D\}, \\
\mathcal{H} & :=\{\exp (h E) \mid h \in \operatorname{ker} E \cap \operatorname{ker} D\} .
\end{aligned}
$$

Remark that $\operatorname{ker} E \cap \operatorname{ker} D=\mathbb{C}[z]$ and thus $\mathcal{H}=\{(x+h, y, z) \mid h \in \mathbb{C}[z]\}$.

Proposition 1. We have a semi-direct product decomposition

$$
\operatorname{Cent}(\mathbf{u})=C \ltimes(\mathcal{H} \ltimes \mathcal{F}) .
$$

Proof. Recall that $p=x z+y^{2}, z \in \operatorname{ker} D$. In fact, $R:=\operatorname{ker} D$ is the polynomial ring $\mathbb{C}[z, p]$ by $\left[1\right.$, Theorem (b)]. We have $R[x]=\mathbb{C}\left[z, x, y^{2}\right]$ and hence a decomposition $\mathbb{C}[x, y, z]=R[x] \oplus y R[x]$. Let $\mathbf{g}=\left(g_{1}, g_{2}, g_{3}\right) \in \operatorname{Cent}(\mathbf{u})$. Write $g_{1}=v+y q$ with polynomials $v, q \in R[x]$. In $\mathbb{C}[x, y, z, t]$ we have, by definition,

$$
v\left(x-2 t y-t^{2} z\right)+(y+t z) q\left(x-2 t y-t^{2} z\right)=v(x)+y q(x)-2 t g_{2}-t^{2} g_{3} .
$$

A comparison of the coefficients with respect to the variable $t$ shows that $v=r+s x$ with $r, s \in R$, and $q \in R$. Hence, we get $g_{1}=r+s x+q y, g_{2}=s y-(q / 2) z, g_{3}=s z$, and $s \in \mathbb{C}^{*}$. Up to post composition with an element of $C$ we can assume that $s=1$. Thus,

$$
\mathrm{g} \circ \exp \left(\frac{q}{2} D\right)=\left(x+r+\frac{q^{2}}{4} z, y, z\right) .
$$

One easily sees that this automorphism belongs to $\mathcal{H}$.

Let $\mathcal{U}$ be the ind-subgroup $\mathcal{H} \circ \mathcal{F} \subseteq \mathcal{G}_{3}$. Every element $\mathbf{g}=\exp (h E) \circ \exp (f D) \in$ $\mathcal{H} \circ \mathcal{F}$ satisfies

$$
\mathbf{g}^{m}=\exp (m h E) \circ \exp \left(\sum_{i=0}^{m-1} \exp (i h E)^{*}(f) D\right) \quad \text { for all } m \in \mathbb{N} .
$$

Since $\mathcal{H} \cap \mathcal{F}=\{\mathbf{i d}\}$, this shows that every element $\neq \mathbf{i d}$ of $\mathcal{U}$ has infinite order and is contained in an algebraic subgroup of $\mathcal{U}$. Thus every element of $\mathcal{U}$ is unipotent. Now, Proposition 1 implies that $\mathcal{U}$ is the set of unipotent elements of Cent( $\mathbf{u})$. A calculation shows that

$$
\mathcal{F}=\operatorname{Cent}_{\mathcal{U}}[\mathcal{U}, \mathcal{U}]
$$

where $[\mathcal{U}, \mathcal{U}]$ is the commutator subgroup of $\mathcal{U}$. Let $T:=\left\{\left(a^{2} b^{-1} x, a y, b z\right) \mid a, b \in \mathbb{C}^{*}\right\}$ which is a closed algebraic subgroup of $\mathcal{G}_{3}$. The torus $T$ normalizes $\operatorname{Cent}(\mathbf{u}), \mathcal{H}, \mathcal{F}$ 
and $\mathcal{U}$. In fact, it follows from equation $(\triangle)$ that $T$ is the largest subgroup of the standard torus in $\mathcal{G}_{3}$ that normalizes $\operatorname{Cent}(\mathbf{u})$.

Proof of the Application. As $\mathbf{u}$ is a tame automorphism, $\theta$ preserves $\operatorname{Cent}(\mathbf{u})$. A calculation shows that the neutral element is the only element of $\mathcal{H}$ (of $\mathcal{F}$ ) that commutes with $T$. Thus the Main Theorem applied to the unipotent ind-subgroups $\mathcal{H}$ and $\mathcal{F}$ of $\mathcal{G}_{3}$ implies that $\theta(\mathcal{U})=\mathcal{U}$. From equation $(\triangle)$ it follows that $\theta$ preserves $\mathcal{F}$. We define $\mathscr{V}_{\mathcal{F}}$ as the set of all algebraic subgroups of $\mathcal{F}$ which are isomorphic to $\mathbb{C}^{+}$and which are normalized by $T$. One can see that the elements of $\mathscr{V}_{\mathcal{F}}$ correspond to the locally nilpotent derivations $z^{m} p^{n} D$ for all $m, n \geq 0$ and hence different elements in $\mathscr{V}_{\mathcal{F}}$ have different characters. This implies that for all $V \in \mathscr{V}_{\mathcal{F}}$ there exists $a_{V} \in \mathbb{C}^{*}$, such that $\left.\theta\right|_{V}=a_{V} \operatorname{id}_{V}$. Let $\mathbf{e}:=(x-1, y, z) \in \mathcal{H}$. A calculation shows that

$$
\mathbf{e} \circ \exp \left(z^{m} p^{n} D\right) \circ \mathbf{e}^{-1}=\exp \left(\sum_{i=0}^{n}\left(\begin{array}{c}
n \\
i
\end{array}\right) z^{i+m} p^{n-i} D\right) \quad \text { for all } m, n \in \mathbb{N} \text {. }
$$

Applying $\theta$ to the last equation and using the fact that $\exp \left(z^{j} D\right)$ and e are tame automorphisms yields $a_{V}=1$ for all $V \in \mathscr{V}_{\mathcal{F}}$. As all the subgroups $V \in \mathscr{V}_{\mathcal{F}}$ generate $\mathcal{F}$, it follows that $\theta$ is the identity on $\mathcal{F}$. Since $\mathcal{H}$ and $C$ consist of tame automorphisms this finishes the proof.

\section{Acknowledgments}

I would like to thank Hanspeter Kraft for many fruitful discussions. I am supported by the Swiss National Science Foundation (Schweizerischer Nationalfonds).

\section{References}

[1] H. Bass, A nontriangular action of $\boldsymbol{G}_{a}$ on $\boldsymbol{A}^{3}$, J. Pure Appl. Algebra 33(1) (1984), 1-5.

[2] H. Bass, E.H. Connell, and D. Wright, The Jacobian conjecture: reduction of degree and formal expansion of the inverse, Bull. Amer. Math. Soc. (N.S.) 7(2) (1982), 287-330.

[3] A. Belov-Kanel and J.-T. Yu, On the Zariski topology of automorphism groups of affine spaces and algebras, 2013, http://arxiv.org/abs/1207.2045.

[4] G. Freudenburg, Algebraic theory of locally nilpotent derivations, Vol. 136 (Encyclopaedia of Mathematical Sciences), Springer-Verlag, Berlin, 2006, ISBN 978-3-540-29521-1; 3-540-29521-6. Invariant Theory and Algebraic Transformation Groups, VII.

[5] J.E. Humphreys, Linear algebraic groups, Graduate Texts in Mathematics, No. 21, SpringerVerlag, New York, 1975.

[6] H.W.E. Jung, Über ganze birationale Transformationen der Ebene, J. Reine Angew. Math. 184 (1942), 161-174.

[7] H. Kraft and I. Stampfli, On Automorphisms of the Affine Cremona Group, Ann. Inst. Fourier (Grenoble) 63(3) (2013), 1137-1148.

[8] S. Kumar, Kac-Moody groups, their flag varieties and representation theory, Vol. 204 of Progress in Mathematics, Birkhäuser Boston Inc., Boston, MA, 2002, ISBN 0-8176-4227-7.

[9] S. Kuroda, Wildness of polynomial automorphisms in three variables (2011), http://arxiv.org/abs/1110.1466.

[10] I.P. Shestakov and U.U. Umirbaev, The tame and the wild automorphisms of polynomial rings in three variables, J. Amer. Math. Soc. 17(1) (2004), 197-227 (electronic).

[11] W. van der Kulk, On polynomial rings in two variables, Nieuw Arch. Wiskunde (3) 1 (1953), $33-41$.

Mathematisches Institut, Universität Basel, Rheinsprung 21, CH-4051 Basel, SWITZERLAND

E-mail address: immanuel.e.stampfli@gmail.com 
